\title{
Narrative Ordnung?
}

\author{
Erzählperspektiven in der Nibelungenklage
}

\section{Einleitung: Fokalisierung und perspektivisches Erzählen}

Inwiefern verhandeln vormoderne Erzähltexte Ordnungsvorstellungen einer Gesellschaft? In welcher Funktion kann die Erzählliteratur beanspruchen, „an der Gestaltung soziokultureller Welt“ Teil zu haben?' ${ }^{1}$ Für das übergeordnete Frageinteresse des Bandes erscheint ein Blick auf die Heldenepik, die „seit jeher als Teil einer Gedächtniskultur verstanden“ wird, ${ }^{2}$ als besonders lohnend. Die darin aufgehobene kollektive Erinnerung als institutionelle Sicherung des Bewahrenswerten bildet ein gemeinschafts- und identitätsstiftendes Potential aus. ${ }^{3}$ Das gleichsam zu einer Text-Ordnung transformierte kollektive Gedächtnis repräsentiert das Selbstverständnis von Gesellschaften und deren Denkweisen von Welt; einer solchen Erinnerungsleistung eignet insofern epistemologische Kraft, weil sie systematisch Wissensordnungen als zentrale Prozesse kultureller Sinnbildung reflektiert. Indem das Weltwissen eines Kollektivs nicht nur sinnstiftend organisiert, sondern auch funktional im Kontinuum von Vergangenheit, Gegenwart und Zukunft verortet wird, stellt es die Grundlage jeder Herrschaftslegitimation bereit. ${ }^{4}$ Dass dabei das Verhältnis von irritierter Gesellschaftsordnung und der

1 Fuhrmann, Daniela/Selmayr, Pia: Ordnen, Wissen, Verstehen. Theoretische Vorüberlegungen. In diesem Band, S. 14.

2 MÜLLER, Jan-Dirk: Nibelungenlied und kulturelles Gedächtnis. In: Arbeiten zur Skandinavistik, 14. Arbeitstagung der Deutschsprachigen Skandinavistik, 1.-5.9.1999 in München. Hrsg. von Annegret Heitmann, Bern 2001, S. 29-43, hier S. 31. Zum historischen Bezug der Heldensage vgl. HEInZLE, Joachim: Die Nibelungensage als europäische Heldensage. In: Die Nibelungen. Sage - Epos - Mythos. Hrsg. von Joachim HeInzLE, Wiesbaden 2003, S. 3-29. Siehe auch KROPIK, Cordula: Reflexionen des Geschichtlichen. Zur literarischen Konstituierung mittelhochdeutscher Heldenepik, Heidelberg 2008 (Jenaer Germanistische Forschungen 24).

3 Vgl. dazu Assmann, Jan: Das kulturelle Gedächtnis. Schrift, Erinnerung und politische Identität in frühen Hochkulturen, München 1992, bes. S. 88.

4 „Herrschaft legitimiert sich retrospektiv und verewigt sich prospektiv“: AssmanN, Gedächtnis, S. 71 (Anm. 3). Vgl. auch OEXLE, Otto G.: Memoria in der Gesellschaft und in der Kultur des Mittelalters. In: Modernes Mittelalter. Hrsg. von Joachim HeInZLE, Leipzig 1994, S. 297-323, hier S. 313.

Dr. Julia Frick, Universität Zürich, Deutsches Seminar, Ältere deutsche Literatur, Schönberggasse 9, 8001 Zürich, julia.frick@ds.uzh.ch

Ә Open Access. (C) 2021 Julia Frick, publiziert von De Gruyter. (cc) BY Dieses Werk ist lizensiert unter einer Creative Commons Namensnennung 4.0 International Lizenz. https://doi.org/10.1515/9783110729115-004 
Ordnung, die diese in ihrer Abbildung zu fordern scheint, ${ }^{5}$ produktiv verhandelt wird, dokumentiert das Ende des Nibelungenliedes als größtmögliche Un-Ordnung, vor der der Erzähler resigniert:

Ine kán iu niht bescheiden, waz sider dâ geschach:

wan ritter unde vrouwen weinen man dâ sach, dar zuo die edeln knehte ir lieben friunde tôt.

hie hât daz mære ein ende: daz ist der Nibelunge nôt. ${ }^{6}$

(Str. 2379)

Doch das ist nur eine Sichtweise, die sich den modernen Editionen verdankt. ${ }^{7}$ Die Lektüre der mittelalterlichen Rezipienten ging über das nicht Erzählbare hinaus, um in der Nibelungenklage aufgehoben zu werden: Dieser Text belässt es nicht bei einer bloßen Fortführung der Handlung, sondern gliedert die Ereignisse in christlich geprägte Denkkategorien ein und bietet insofern Ordnungsmuster für die Bewältigung von Trauer und Tod. In der Tradition des Planctus wird dem Tod als das allen Menschen bevorstehende Schicksal (communis hominum condicio) sein Schrecken genommen: ${ }^{8}$

\footnotetext{
man muoz die varen lâzen, die uns tägelîch nimt der tôt. daz ist ein gewonlîch nôt, swâ man daz von im vernimt, wan im anders niht enzimt,
}

5 Vgl. Fuhrmann/Selmayr, Ordnen, Wissen, Verstehen, S. 20-25 (Anm. 1).

6 Zitate hier wie im Folgenden nach: Das Nibelungenlied. Nach dem Text von Karl BARTSCH und Helmut DE BOOR ins Neuhochdeutsche übersetzt und kommentiert von Siegfried GROSSE, Stuttgart 2003.

7 Einen Fortschritt gegenüber der bisherigen Editionspraxis bietet die Ausgabe: Das Nibelungenlied und die Klage. Nach der Handschrift 857 der Stiftsbibliothek St. Gallen. Mittelhochdeutscher Text, Übersetzung und Kommentar. Hrsg. von Joachim HeInZLE, Berlin 2015 (Deutscher Klassiker-Verlag im Taschenbuch 51).

8 Vgl. dazu von Moos, Peter: Consolatio. Studien zur mittellateinischen Trostliteratur über den Tod und zum Problem der christlichen Trauer. 4 Bde., München 1971/72. Die Nähe der Nibelungenklage zum lateinischen Planctus erscheint umso augenfälliger, nicht nur weil sie sich in der Selbstbezeichnung der Dichtung im Schlussvers (Dizze liet heizet diu Klage. ${ }^{\star} \mathrm{C}$ V. 4428) manifestiert, sondern weil der Gleichheitstopos als „Hauptgegenstand der ,Anklage“ im Planctus“ (von Moos, Consolatio, Testimonienband, S. 115, T 552) angesichts des Todes Eingang gefunden hat: Christen und Heiden ( ${ }^{\star}$ B V. 1849f.), tumbe und wîse, tôren und grîse ( ${ }^{\star} \mathrm{B}$ V. 1950-1953) klagen gemeinsam um die Toten. 
wan daz er liep von liebe schelt, unz er uns alle hin gezelt.

der tôt anders niht enkan. ${ }^{9}$

$\left({ }^{\star}\right.$ C V. 3540-3547)

Die Nibelungenklage lässt sich hinsichtlich ihrer Gattungszugehörigkeit gewissermaßen als Hybride bezeichnen: ${ }^{10}$ Dem Stoff nach bietet sie heroische Überlieferung, jedoch wird das Idiom des Nibelungenliedes aufgegeben zugunsten des Reimpaarverses des höfischen Romans, der die „Abwendung von der Tradition “ unterstreicht und damit einen Gattungswechsel anzeigt. ${ }^{11}$ Die Forschung hat sich mit der literarisch-qualitativen, nicht minder aber mit der ästhetischen Einordnung dieses Textes, die stets in Bezug auf das Nibelungenlied erfolgte, außerordentlich schwergetan: ${ }^{12}$ Signum der „schwache[n] Dichtung“ sei die „Langwierigkeit des mageren Geschehens“, vor allem aber „der mechanisch-hölzerne Tonfall seiner spannungslosen Verse“; $;^{13}$ als „sehr mittelmäßiges Werk ${ }^{\text {114 }}$ und „Dichtung von künstlerisch-ästhetisch geringem Rang،15 erreiche sie das als stilistisch höherwertig betrachtete Nibelungenlied nicht; es handle sich vielmehr um eine ,überflüssige, um nicht zu sagen unerwünschte Fortsetzung des ,Nibelungenliedes“““ ${ }^{16}$ So erschien die Nibelungenkage im Urteil

9 Die Nibelungenklage wird hier und im Folgenden zitiert nach: Die ,Nibelungenklage‘. Synoptische Ausgabe aller vier Fassungen. Hrsg. von Joachim BuMKE, Berlin, New York 1999.

10 Zur Gattungsdiskussion siehe SzKLENAR, Hans: Die literarische Gattung der Nibelungenklage und das Ende „alter maere“. In: Poetica 9 (1977), S. 43-49, und die Forschungsübersicht bei DECK, Monika: Die Nibelungenklage in der Forschung. Bericht und Kritik, Frankfurt a. M. 1996, S. 187-197.

11 MÜLLER, Jan-Dirk: ,Episches‘ Erzählen. Erzählformen früher volkssprachiger Schriftlichkeit, Berlin 2017 (Philologische Studien und Quellen 259), S. 154.

12 Zur Forschungsgeschichte vgl. BUMKE, Joachim: Die vier Fassungen der ,Nibelungenklage‘. Untersuchungen zur Überlieferungsgeschichte und Textkritik der höfischen Epik im 13. Jahrhundert, Berlin, New York 1996 (Quellen und Forschungen zur Literatur- und Kulturgeschichte 8 [242]), S. 104 f. Siehe auch Nibelungenlied und Nibelungensage. Kommentierte Bibliographie 1945-2010. Hrsg. von Florian KRAGL, bearb. von Elisabeth MARTSCHINI, Berlin 2012.

13 WeHRLI, Max: Die „Klage“ und der Untergang der Nibelungen. In: Zeiten und Formen in Sprache und Dichtung. FS für Fritz Tschirch zum 70. Geburtstag. Hrsg. von Karl-Heinz SCHIRMER/ Bernhard Sowinski, Köln, Wien 1972, S. 104.

14 DE BooR, Helmut: Die höfische Literatur. Vorbereitung, Blüte, Ausklang. 11. Aufl. bearb. von Ursula HenNIG, München 1991 (DE BOOR-NEWALD: Geschichte der deutschen Literatur von den Anfängen bis zur Gegenwart 2), S. 167.

15 VooRwinden, Norbert: Nibelungenklage und Nibelungenlied. In: Hohenemser Studien zum Nibelungenlied. Unter Mitarbeit von Irmtraud ALBRECHT. Hrsg. von Achim MASSER, Dornbirn 1981, S. 276.

16 ÖHMAnN, Emil: Anklänge an Ulrichs von Zazikhoven Lanzelet in Nibelungenlied, Nibelungenklage und Wigalois. In: Neuphilologische Mitteilungen 47 (1946), S. 61-82, hier S. 70. 
der Forschung lange Zeit als defizitärer, unterkomplexer Text, dem allenfalls ein

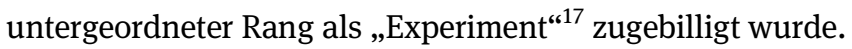

Dass die materiale Beschaffenheit der Überlieferung einen von dieser Sichtweise abweichenden Befund bietet, ist hinreichend bekannt: Die enge Koppelung von Nibelungenlied und Klage wird durch die formale Einrichtung des Textes, nämlich das „Bemühen um graphische Angleichung der beiden Teile“, ${ }^{18}$ angezeigt, indem die Reimpaarverse so organisiert sind, dass sie optisch den Strophen gleichkommen. ${ }^{19}$ Der Textverbund dürfte also für die mittelalterlichen Rezipienten - zumindest jedenfalls für die Schreiber - ein geschlossenes Gefüge, das sog. „Nibelungen-Buch“, ${ }^{20}$ gebildet haben. In dieser Konzeption erscheint das Nibelungengeschehen, das in der Klage bis zur Stiftung der neuen Landesherrschaft in Worms weitergeführt und aus den christlich geprägten Denkkategorien um 1200 heraus gedeutet wird, als ,komplettiert'. Diese Verankerung der Ereignisse im geistigen Rahmen der Zeit verleiht der Dichtung die Aura einer legitimen Sinnstiftung für das Nibelungengeschehen. Und da die Klage das gesamte Mittelalter hindurch die einzige Deutung bleibt, gibt es keinen Grund, den durch die Überlieferung dokumentierten „sinnstiftende[n] Konnex“21 und damit die Funktion der Klage als akzeptierte und vielleicht sogar als notwendig erachtete Sinngebung nicht ernst zu nehmen. ${ }^{22}$

Die mit diesen Überlegungen umrissene Ebene der erzählten Ordnung in der Nibelungenklage ist mit spezifischen Ordnung(en) des Erzählens verknüpft, die in ein sinnstiftendes Verhältnis zueinander treten. Aufgrund des markanten Charakters des Textes als narrative ,Bewältigung، der von den Figuren erlebten Ereignisse wird die Handlung zu einem nicht unerheblichen Teil durch Kommentare verschiedener Stimmen über das Nibelungengeschehen vorangetrieben. Dieses Phänomen ist in der Forschung schon früh beobachtet worden: Michael CURSCHMANN hat die in der Nibelungenklage vorliegende „Vielzahl der

17 WACHINGER, Burghart: Die Klage und das Nibelungenlied. In: Hohenemser Studien zum Nibelungenlied (Anm. 15), S. 266.

18 BumKe, Die vier Fassungen, S. 237 (Anm. 12).

19 Man muss „schon genau hinsehen und dabei auf die Reimstellungen achten [...], um festzustellen, ob man eine ,Lied'-Seite oder eine ,Klage'-Seite vor sich hat“. Ebd., S. 239.

20 Vgl. HeinZle, Joachim: Die Handschriften des Nibelungenliedes und die Entwicklung des Textes. In: Die Nibelungen. Sage - Epos - Mythos. Hrsg. von Joachim HeInzLE, Wiesbaden 2003, S. 191-212, hier S. 198.

21 HENKEL, Nikolaus: Nibelungenlied und Klage. Überlegungen zum Nibelungenverständnis um 1200. In: Mittelalterliche Literatur und Kunst im Spannungsfeld von Hof und Kloster. Ergebnisse der Berliner Tagung, 9.-11. Oktober 1997. Hrsg. von Nigel F. PALMER/Hans-Jochen SCHIEWER, Tübingen 1999, S. 73-98, hier S. 83.

22 Vgl. ebd., S. $97 \mathrm{f}$. 
internen Stimmen“ als eine ,interne[] Diskussion um die Gründe und die Schuld an der Tragödie“ gesehen, ,die durchaus nicht in allem zu einheitlichen Ergebnissen kommt.“23 Damit bilde die Nibelungenklage eine „lebendige[] und vielgestaltige[] Erzähltradition“ ab und projiziere „das Schema vielstimmiger ,Vergangenheitsbewältigung` zurück in eine selbst schon ,vielstimmige‘ Stofftradition“ ${ }^{24}$ Das setzt perspektivisches Erzählen voraus, auch wenn damit bei CURSCHMANN nicht primär die narrative Technik erfasst, sondern der durch den Überlieferungsprozess bedingte Status des Textes als „,vielgestaltiges historisches Phänomen “ ${ }^{\star 25}$ zwischen Mündlichkeit und Schriftlichkeit beschrieben wird. Elisabeth LIENERT hingegen argumentiert explizit gegen grundsätzlich verschiedene narrative Perspektiven im Erzählverfahren der Klage und sieht allenfalls das alii dicunt-Prinzip gelehrter Chronistik als Vorbild: „Echte Mehrstimmigkeit freilich liegt nicht vor: Alle sagen dasselbe; der Erzähler vervielfacht im Grunde sich selbst. “26

Jüngere Ansätze unterscheiden zwei genrespezifische Ausprägungen der narrativen Technik der Erzählperspektive: Einerseits postuliert Jan-Dirk MÜLLER eine a-perspektivische Einstimmigkeit für das ,epische“ Erzählen, das „eine Aufspaltung von Erzählpositionen [...] nicht“" kenne. ${ }^{27}$ Die Möglichkeit für fokalisiertes Erzählen sei zwar grundsätzlich gegeben; die Multiplizierung von Erzähler- und Figurenstimmen bringe jedoch nicht ,echte', standpunktabhängige Mehrstimmigkeit hervor, sondern habe die Funktion, „Konsens zu zelebrieren“. ${ }^{28}$ Die auf eine Homogenisierung der Weltsicht zielende Aufsplitterung der Stimmen bilde ein genuines Merkmal der Gattung noch in der spätmittelalterlichen Heldenepik: „Die wechselnden Fokalisierungen stellen die Einstimmigkeit der Aussage nicht in Frage, sondern stützen sie. Indem im Prinzip alle dasselbe sagen, verschmelzen die vielen Stimmen zu einer einzigen.“29

23 CURSCHMANN, Michael: Nibelungenlied und Nibelungenklage. Über Mündlichkeit und Schriftlichkeit im Prozeß der Episierung. In: Deutsche Literatur im Mittelalter - Kontakt und Perspektiven. Hugo Kuhn zum Gedenken. Hrsg. von Christoph CoRMEAU, Stuttgart 1979, S. 85-119; wiederabgedruckt in: Nibelungenlied und Nibelungenklage. Neue Wege der Forschung. Hrsg. von Christoph Fasbender, Darmstadt 2005, S. 160-190, hier S. $173 \mathrm{f}$.

24 Ebd., S. 173 u. 175.

25 Ebd., S. 175.

26 Die Nibelungenklage. Mittelhochdeutscher Text nach der Ausgabe von Karl Bartsch. Einführung, neuhochdeutsche Übersetzung und Kommentar von Elisabeth LIENERT, Paderborn 2000, S. 41.

27 MÜLLER, ,Episches‘ Erzählen, S. 211 (Anm. 11).

28 Ebd., S. 214.

29 Ebd., S. 241. 
Andererseits hat Gerd HÜBNER nachgewiesen, dass punktuell realisierte, durch unterschiedliche Fokalisierungsinstanzen vermittelte subjektive Positionen $\mathrm{zu}$ den wesentlichen Elementen romanhaften Erzählens gehören. ${ }^{30}$ Die „Filterung der narrativen Information auf einen Figurenhorizont hin“"31 verwische „die Grenze zwischen der Erzählerrede und den Wahrnehmungen, Realitätskategorien und Reflexionen der Figur“ “. ${ }^{32}$ Damit sei zwar kein grundsätzlich differenter Standpunkt zwischen Erzähler und Figuren markiert; gleichwohl ziele der als subjektiver Modus inszenierte Nachvollzug alternativer Normhorizonte darauf, die evaluativen Begründungssprachen als standpunktabhängig erscheinen $\mathrm{zu}$ lassen und so „die Existenz des Gegenstandpunktes“ erfahrbar zu machen. ${ }^{33}$ Insofern ermögliche die Funktion der Fokalisierungstechnik Einsichten in die „Relativität von Verhalten und Urteil“" 34

Gerade die textsortenspezifische Zugehörigkeit der Nibelungenklage zum Typus des Planctus erlaubt eine Vervielfältigung der Stimmen. In der Zuweisung von „Redeanteilen einerseits an den Erzähler, andererseits an die Figuren““35 lassen sich spezifische Möglichkeiten narrativer Sinnstiftung beobachten, die das Geschehen als subjektive Wahrnehmung der einzelnen Figuren fokalisieren. Betrachtet man die Kategorie der Erzählperspektive unter Berücksichtigung der Eigenheiten mittelalterlichen Erzählens - wie dies Brigitte BURRICHTER plausibilisiert - als komplexes Ineinander von Erzählstimme, die eine jeweils ,sprecherabhängige' Positionierung zum erzählten Geschehen fokussiert, und Fokalisierung, die die Informationsvergabe mittels der fokalisierten Instanzen selbst steuert, ${ }^{36}$ lässt sich dieses Konzept produktiv auf die Ordnung des Erzählens in der Nibelungenklage anwenden. Perspektivisches Erzählen wäre in diesem Sinne als ein bestimmter Typus der Fokalisierungstechnik zu fassen, der nicht allein mit dem Wechsel verschiedener Stimmen und Standpunkte, sondern mit verschiedenen Deutungsangeboten operiert, mit „evaluativen Kommentare[n], ${ }^{37}$

30 Vgl. HÜBNER, Gert: Erzählform im höfischen Roman. Studien zur Fokalisierung im Eneas, im Iwein und im Tristan, Tübingen, Basel 2003 (Bibliotheca Germanica 44).

31 Ebd., S. 393.

32 Ebd., S. 199.

33 Ebd., S. 200 u. 201.

34 Ebd., S. 200.

35 MÜLLER, ,Episches‘ Erzählen, S. 199 (Anm. 11).

36 Zur Interdependenz von Erzählstimme, Erzählperspektive und Fokalisierung vgl. in historischem Zugriff BuRRICHTER, Brigitte: Perspektive - Mittelalter. In: Handbuch historische Narratologie. Hrsg. von Eva von ConTZEN und Stephan TiLG, Stuttgart 2019, S. 149-156.

37 HÜBNER, Erzählform, S. 98 (Anm. 30). 
die eine „unaufhebbare[] Diversität von Weltsichten“, ${ }^{38} \mathrm{~d}$.h. eine explizite und unentscheidbare Konkurrenz von Wertungsparametern dokumentieren.

Vor dem Hintergrund dieser Überlegungen ist nach der Art und Weise der Funktionalisierung unterschiedlicher Erzählperspektiven in der Nibelungenklage zu fragen: Generieren die Erzählinstanzen, deren evaluative Kommentare das Weitererzählen der Nibelungenhandlung konstituieren, tatsächlich perspektivisches Erzählen? Wie sind die Effekte der mit der Fokalisierung verbundenen Informationsvergabe einzustufen? Als narrative Nuancierungen einer einstimmigen und damit ,einsinnigen' Erzählordnung oder als Repräsentationsmomente grundsätzlich verschiedener Wertungs- und Deutungspotenziale? Die Annäherung erfolgt in drei Schritten: Denkordnung(en) von Schuld und Sühne (II.); der Botenbericht als metadiegetische Erzählordnung (III.); historische Diskursordnung: Fassungsdivergenz (IV.). Das Fazit bietet die Sondierung der Ergebnisse und die Beantwortung der Frage (V.).

\section{Die Denkordnung(en) von Schuld und Sühne}

Die Beantwortung der Schuldfrage ist zentrales Anliegen der Nibelungenklage. Die Erzählerstimme etabliert in den ersten rund 600 Versen eine allgemein verbindliche Werte- und Deutungsordnung, die programmatisch auf die Lektüre des in der materialen Überlieferung vorausgehenden Textes ausgerichtet ist.

Warum muss Siegfried sterben? Der Erzähler führt Siegfrieds übermuot als Ursache für seinen Tod ein (und daz er selbe den tôt / gewan von sîner übermuot ${ }^{\star}$ B V. 38f.) und rekurriert damit auf den aus dem Nibelungenlied vertrauten Habitus des Helden. ${ }^{39}$ Gleichwohl verurteilt er die Ausführenden, Kriemhilds nächste Verwandte ( ${ }^{\star} B$ V. 80 f.), für ihre Tat:

dem [i. e. Siegfried] vil übel an gewan
ir bruoder Gunthêr den lîp
und Hagen und des küneges wîp,
von dem er doch den tôt genam.
dem helde sterben niht gezam
von deheines recken hant [...]

$\left({ }^{\star}\right.$ B V. 102-107)

38 MÜLLER, ,Episches‘ Erzählen, S. 200 (Anm. 11).

39 Vgl. MÜLLER, Jan-Dirk: Spielregeln für den Untergang. Die Welt des Nibelungenliedes, Tübingen 1998, S. 237. Vgl. z. B. Kriemhilds Aussage gegenüber Hagen: ob er [i. e. Siegfried] nicht volgen wolde sîner übermuot; / sô waere immer sicher der degen küene unde guot (Str. 896, 3-4). 
Der Hinweis auf den unwürdigen Tod Siegfrieds rekurriert auf eine andere Bedeutungsnuance des mhd. Lexems übermuot, nämlich die mit dem Begriff der superbia belegte „verdammenswerte Selbstüberhebung des Menschen“40 als initium omnis peccati $(\mathrm{Ecl} 10,15)$. Sie manifestiert sich in der Bezeichnung Hagens als übermüete hêre ( ${ }^{\star} \mathrm{B}$ V. 230). Daneben lässt sich die Schuld der Burgunden auf die Sünde der avaritia zurückführen (daz Kriemhilde golt rôt / heten si ze Rîne lâzen *B V. 192f.). Die Erzählerstimme verweist daher auf den Tod als gerechte Strafe für ihre Vergehen: ich waene, si ir alten sünde / engulten und niht mêre ( ${ }^{\star} \mathrm{B}$ V. 196f.).

Auf dieser argumentativen Basis wird der Tod der Burgunden als unausweichliches Schicksal und gerechte Strafe Gottes am urteillîch tac ( ${ }^{\star} \mathrm{B}$ V. 216) verstanden: dô muos in misselingen / von einen alten schulden ( ${ }^{\star} \mathrm{B}$ V. 226f.); exemplarisch heißt es von Gunther:

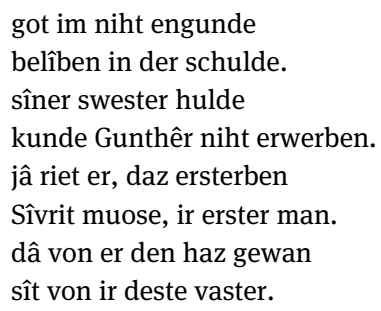

(*B V. 490-497)

Durch die Figurenstimmen wird besonders Hagen als Schuldiger exponiert: So bezeichnet Hildebrand ihn bei der Auffindung seines Leichnams als vâlant / [...] der ez allez riet ( ${ }^{\star} \mathrm{B}$ V. 1250f.); ${ }^{41}$ die Formulierung kontrastiert mit der Darstellung Kriemhilds im Nibelungenlied als vâlandinne (Str. 2371,4). Allein Hagen sei es anzulasten, daz manz mit güete niene schiet ( ${ }^{\star} \mathrm{B}$ V. 1252). Aus diesem Grund deutet Hildebrand, im Einklang mit der Erzählerstimme, den Nibelungenuntergang als gerechte Strafe Gottes:

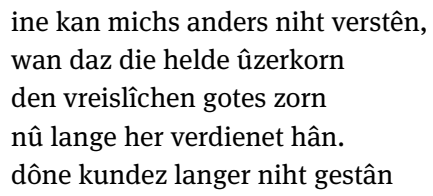

40 HENKEL, Nibelungenlied und Klage, S. 87 (Anm. 21).

41 Die Stimmen der Landbevölkerung reproduzieren diese Wertung: Hagen habe mit seiner vâlandes hant $\left({ }^{\star} \mathrm{C}\right.$ V. 1379) viel Unheil angerichtet. Auch die Schaulustigen auf Swämmels Reise verweisen auf seinen übermuot $\left({ }^{\star} \mathrm{B}\right.$ V. $3525 /{ }^{\star} \mathrm{C}$ V. 3599$)$. 
über ir zil einen tac.

dô muosen si den gotes slac

lîden durch ir übermuot.

$\left({ }^{\star}\right.$ B V. 1270-1277)

Bischof Pilgrim übernimmt die christliche Deutungsperspektive:

der Nibelunge golt rôt,

heten si daz vermiten,

sô möhten si wol sîn geriten

zir swester mit ir hulden.

(*B V. 3430-3433)

Darum traf sie der Tod von ir selber schulden / und von ir starken übermuot ( ${ }^{\star}$ B V. $3434 \mathrm{f}$.). Diese Begründungssprache aktualisiert auch Rumold, der im Nibelungenlied von der Reise ins Hunnenreich abgeraten hatte: mîne herren, die hân ich verlorn / niwan von Hagenen übermuot ( ${ }^{\star} \mathrm{B}$ V. $4030 \mathrm{f}$.). Dessen Schuld, der Mord an Siegfried sowie der Hortraub (dô er Kriemhilde nam ir man / und ir ir guot an gewan ${ }^{\star} \mathrm{B}$ V. 4033f.), markieren die Sünde der superbia: des er niht tuon solde / [...] wande des was im gar ze vil ( ${ }^{\star} \mathrm{B}$ V. 4043-4045).

Mit der eindeutigen Schuldzuweisung an Hagen und den burgundischen Personenverband geht die Erklärung und Motivierung von Kriemhilds Handeln mittels der Kategorie der triuwe einher. Während im Nibelungenlied die Ambiguisierung der triuwe an der Rüdiger-Figur durchgespielt wird, ${ }^{42}$ aber auch Kriemhilds „radikal individualisiert[e], nurmehr auf eine Person gerichtet[e]“43 triuwe als gleichermaßen ambivalent erscheint, da sie sämtliche sozialen Rücksichten ausblendet, ist es Forschungskonsens, dass die Nibelungenklage den Wert der triuwe positiviert und damit Kriemhild von der Verantwortung für den Nibelungenuntergang entlastet: Die Klage mache „die Ambiguisierung von triuwe rückgängig“ “44 die als Wert von sozialem Gehalt im Lied problematisiert werde.

42 Der Markgraf „erfährt den Widerspruch zwischen unterschiedlichen Verpflichtungen als Zerstörung seiner Existenz“. MüLlER, Jan-Dirk: Das Nibelungenlied, Berlin 2009, S. 110. Zu Rüdigers Dilemma vgl. HASEBRINK, Burkhard: Aporie, Dialog, Destruktion. Eine textanalytische Studie zur 37. Aventiure des Nibelungenliedes. In: Dialoge. Sprachliche Kommunikation in und zwischen Texten im deutschen Mittelalter. Hrsg. von Nikolaus Henkel/Martin H. JoNES/Nigel F. PALMER, Tübingen 2003, S. 7-20.

43 MÜLLER, Spielregeln, S. 167 (Anm. 39).

44 Ebd., S. 170. 
Kriemhilds über den Tod hinausreichende triuwe $\mathrm{zu}$ Siegfried veranlasst den Erzähler der Klage zu einem Exkurs, der die triuwe als allgemein verbindlichen ethischen Maßstab einführt: ${ }^{45}$

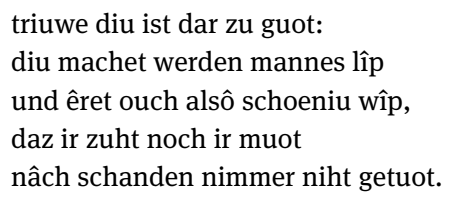

In einem zweiten Schritt wird die solchermaßen als undiskutierter „Höchstwert“46 definierte triuwe Kriemhild zugeordnet. Jeder verständige Mensch müsse sie von aller Schuld freisprechen:

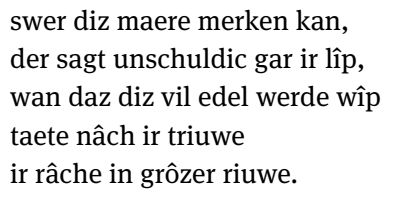

Kriemhilds Rache sei rechtmäßig erfolgt, da sie aufgrund ihrer triuwe-Bindung zu Siegfried nicht anders habe handeln können:

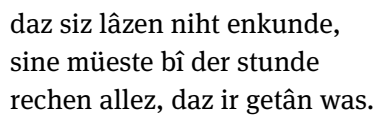

$\left({ }^{\star}\right.$ B V. 231-233)

Denn, so heißt es explizit, ez ir rechen gezam ( ${ }^{\star} \mathrm{B}$ V. 138). Dass die Vergeltung allerdings eine andere Wendung genommen habe, als ursprünglich geplant (daz niwan der eine man [i. e. Hagen] / den lip hete verlorn ${ }^{\star} \mathrm{B}$ V. 262f.), wird nicht Kriemhild, sondern den unglücklichen Umständen zugeschrieben, da sie als Frau nicht in der Lage gewesen sei, sich eigenhändig zu rächen, wie sie das als Mann zu tun vermocht hätte ( ${ }^{\star} \mathrm{B}$ V. $\left.128-133\right) .{ }^{47}$

45 Vgl. dazu Zimmermann, Günter: Der Krieg, die Schuld und die Klage. In: Helden und Heldensage. Otto Gschwantler zum 60. Geburtstag. Hrsg. von Hermann ReICHERT/Günter ZIMMERMANN, Wien 1990, S. 513-536, bes. S. 523.

46 MỦLlER, Spielregeln, S. 168 (Anm. 39).

47 Die Begründung dieses Umstands durch den Erzähler mit dem Verweis auf Kriemhilds kranke[] sinne ( ${ }^{\star} \mathrm{B}$ V. 243) und lützel wîbes $\sin \left({ }^{\star} \mathrm{C}\right.$ V. 2004) entspricht der mittelalterlichen Auffassung von der Torheit als der „Domäne der Frau“. HenKel, Nibelungenlied und Klage, S. 88 
Der positive Wert der triuwe fungiert in der Fokalisierung durch die Erzählerfigur „als alleiniges und bis zum Schluß bestehendes Movens für Kriemhilt““. ${ }^{48}$ Deshalb kann die Erzählerstimme den Gerüchten, die Königin müsse aufgrund ihrer Schuld der helle swaere ( ${ }^{\star} \mathrm{B}$ V. 558) dulden, die auf der Bibel basierende sentenzhafte Wendung entgegenhalten: „dem getriuwen tuot untriuwe wê“ (^B V. 570). ${ }^{49}$ Insofern sei Kriemhild das Himmelreich sicher:

sît si durch triuwe tôt gelac,

in gotes hulden manegen tac

sol si ze himele noch geleben.

$\left({ }^{\star}\right.$ B V. 571-573)

Die sentenzhaft anmutenden Verweise dienen dazu, die durch die Erzählinstanz fokalisierte Begründungssprache nicht als subjektive Perspektive erscheinen zu lassen, sondern einen verbindlichen evaluativen Kommentar zu schaffen. ${ }^{50}$ Die Verweise auf Kriemhilds Schuld (daz leit und daz ungemach / het geprüevet ir selber munt ${ }^{\star} \mathrm{B}$ V. 510 f.) widersprechen dem nicht, sondern werden durch den Deutungshorizont der triuwe relativiert und überdacht.

Die durch die Erzählinstanz konstituierte Begründungssprache wird allerdings nur von einer Figur aufgenommen: Bei der Auffindung von Kriemhilds Leichnam spricht Etzel ein (topisches) Lob über seine Frau aus: getriuwer wîp wart nie geborn ( ${ }^{\star} \mathrm{B}$ V. 834), problematisiert aber gleichzeitig den Wert ,absoluter' triuwe: Dass er im Wissen um Kriemhilds ganze[] triuwe ( ${ }^{\star} \mathrm{B}$ V. 830) lieber alle Länder verlassen hätte, dokumentiert deren zerstörerisches und damit tendenziell negatives, ja gar gefährliches Potential. Geradezu als triuwelôs erscheint Kriemhild in der Unterredung zwischen Swämmel und Rüdigers Tochter Dietlind in Bechelaren. Darauf wird noch zurückzukommen sein.

Weil der Aspekt der Rechtmäßigkeit der Rache auf der Grundlage von Kriemhilds triuwe in den weiteren Figurenreden keine Zustimmung erfährt, dient die Fokalisierungsfunktion offenbar nicht dazu, einen in Erzähler- und

(Anm. 21). Zur weiblichen und männlichen Gewaltausübung vgl. LIENERT, Elisabeth: Geschlecht und Gewalt im Nibelungenlied. In: ZfdA 132 (2003), S. 3-23.

48 Henkel, Nibelungenlied und Klage, S. 92 (Anm. 21).

49 abominantur iusti virum impium et abominantur impii eos qui in recta sunt via (Prv 29,27; „Die Gerechten verabscheuen den gottlosen Menschen und die Gottlosen verwünschen diejenigen, die auf dem rechten Weg sind“). Vulgata-Zitate hier wie im Folgenden nach: Biblia Sacra Iuxta Vulgatam Versionem. Recensuit et brevi apparatu critico instruxit Robert WEBER, editionem quintam emendatam retractatam praeparavit Roger GRYSON, Stuttgart 2007. Vgl. dazu VOORWINDEN, Nibelungenklage und Nibelungenlied, S. 105 (Anm. 15).

50 Vgl. z. B. swes lîp mit triuwen ende nimt, / daz der zem himelrîche zimt ( ${ }^{\star} \mathrm{B}$ V. 575 f.). 
Figurenstimmen konsonanten evaluativen Kommentar $\mathrm{zu}$ inszenieren. Bischof Pilgrim erscheint die Rache nur dann legitim, wenn sie allein Hagen getroffen hätte:

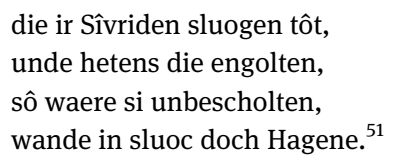

$\left({ }^{\star} \mathrm{B}\right.$ V. 3414-3417)

Diese Aussage nimmt den bereits zuvor vom Erzähler erörterten Sachverhalt auf: mit Hagens Tod waere ir [i. e. Kriemhilds] swaere und ir zorn / [...] gar verswunden ( ${ }^{\star} \mathrm{B}$ V. $264 \mathrm{f}$.); ${ }^{52}$ zugleich bringen die Figurenstimmen die Kategorie des zorns als Begründungssprache für Kriemhilds Handeln ins Spiel, die eine auffallende Differenz zur Fokussierung des Erzählers auf die triuwe hin bildet: Kriemhilds alte[r] zorn ( ${ }^{\star} \mathrm{B}$ V. 918) gegen die Burgunden ist Gegenstand von Etzels Klage bei der Auffindung seines Bruders Bloedelin; Dietrich von Bern sieht als Ursache für Gunthers Tod sîner swester zorne ( ${ }^{\star} \mathrm{B}$ V. 1209); auch Dietlinds Frage, wie Kriemhild Gunther und Hagen empfangen habe (ob si noch iht sêre / zurnde hin zin beiden? ${ }^{\star} \mathrm{B}$ V. 3022f.) markiert das Wissen der Figuren um Kriemhilds Movens für die Rachehandlung.

Diesem Panorama unterschiedlicher Stimmen ist der Botenbericht Swämmels als zentraler Fokalisierungsinstanz an die Seite zu stellen, bevor ein Zwischenresümee erfolgt.

\section{Der Botenbericht als metadiegetische Erzählordnung}

Dem Spielmann als Boten kommt eine besondere Rolle $z u .^{53}$ Als Augenzeuge, dessen Bericht Bischof Pilgrim als Basis für die fingierte Aufzeichnung des Nibelungengeschehens dient, ist er Garant von Wahrheit; sein Bericht repräsentiert

51 Deshalb habe Kriemhild ihre Gäste zu Unrecht vil übele enpfangen ( ${ }^{\mathrm{B}}$ V. 3408).

52 An einer späteren Stelle spricht der Erzähler zwar auch von Kriemhilds zorn, wenn er beklagt, dass so viele Helden von eines wîbes zorne ( ${ }^{\star} \mathrm{B}$ V. 319) gefallen seien. In der durch den Erzähler fokalisierten Begründungssprache spielt der zorn jedoch nur eine untergeordnete Rolle.

53 Zum Botenbericht einschlägig (am Beispiel der gr. Tragödie, aber mit grundsätzlichen Implikationen) schon DE JONG, Irene J. F.: Narrative in Drama. The art of the Euripidean messenger-speech, Leiden 1991 (Mnemosyne Supplementum 116). 
„heroische Überlieferung in statu nascendi““54 und sichert den verbindlichen Anspruch der im Medium der (wohlgemerkt) lateinischen Sprache niedergelegten Überlieferung, wie der Epilog der Nibelungenklage dokumentiert: ${ }^{55}$

Von Pazzouwe der bischof Pilgrîn

durch liebe der neven sîn

hiez schrîben diz maere,

wie ez ergangen waere,

in latînischen buochstaben,

daz manz vür wâr solde haben,

swerz dar nâch ervunde,

von der alrêsten stunde,

wie ez sich huop und ouch began

und wie ez ende gewan

umbe der guoten knehte nôt,

und wie si alle gelâgen tôt.

daz hiez er allez schrîben.

ern liez ez niht belîben,

wand im seit der videlaere

diu kuntlîchen maere,

wie ez ergie und geschach,

wand erz hôrte unde sach [...]

(`B V. 4295-4312)

Swämmel wird als Legitimierungsinstanz der erzählten historia inszeniert, die er an das kollektive Gedächtnis übermittelt: Sein mündlicher Bericht bildet die „Basis einer authentischen schriftlichen Aufzeichnung“56 und erhält dadurch eine wahrheitsverbürgende Funktion. Als erzähltes Ereignis ist er auf einer intradiegetischen Ebene der Erzählung zu verorten; er eröffnet, von der Ausgangsebene der Rahmenhandlung aus betrachtet, eine metadiegetische Dimension. ${ }^{57}$ Das ist deshalb bedeutsam, weil der in Bechelaren und Worms intern fokalisierte Augenzeugenbericht, der als Spiegelung des Rahmenerzählers angelegt ist, in Konkurrenz zur von der Erzählerstimme zu Beginn der Dichtung präsentierten Deutung tritt.

54 MÜLLER, Jan-Dirk: Der Spielmann erzählt. Oder: Wie denkt man sich das Entstehen eines Epos? In: Erzählungen in Erzählungen. Phänomene der Narration in Mittelalter und Früher Neuzeit. Hrsg. von Harald HAFERLAND/Michael MeCKLENBURG, München 1996, S. 85-98, hier S. 95.

55 Zur umstrittenen Deutung der Angabe in latînischen buochstaben zwischen Legitimationstopos und genereller Schriftlichkeitsfiktion vgl. ScHMID, Florian M.: Die Fassung ${ }^{\star} \mathrm{C}$ des Nibelungenlieds und der Klage, Berlin, Boston 2018 (Hermaea N. F. 147), S. $92 \mathrm{f}$.

56 MÜLLER, ,Episches‘ Erzählen, S. 156 (Anm. 11).

$57 \mathrm{Zu}$ dieser Erzähltechnik vgl. MARTínEZ, Matías/SCHEFFEL, Michael: Einführung in die Erzähltheorie. 10. überarbeitete Auflage, München 2016, S. $78 \mathrm{f}$. 
In Bechelaren wird die Schuld am Burgundenuntergang mithilfe der Fokalisierungsinstanzen eindeutig Kriemhild zugewiesen. Die anfangs von Dietrich von Bern angeregte Täuschung über den wahren Ausgang der hôhzit misslingt, ${ }^{58}$ die Wahrheit bricht sich Bahn. Im Wissen über den Tod ihres Vaters durch dessen Gast Gernot spricht Dietlind Kriemhild jegliche triuwe ab:

jâ was ez in beiden [i. e. Gernot, Rüdiger]

nicht guot, daz iemen scheiden

si mit râte solde,

der triuwe haben wolde.

( B V. 3189-3192)

Swämmel bestätigt ihre Perspektive mit einer eindeutigen Stellungnahme:

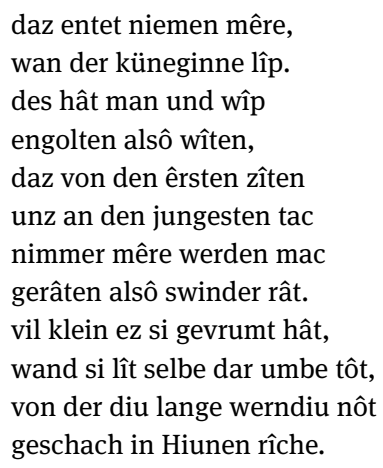

$\left({ }^{\star}\right.$ B V. 3194-3205)

Aber nicht nur in Bechelaren, sondern gerade in Worms dient die Fokalisierungsfunktion dazu, die zu Beginn des Textes durch die Erzählstimme eingeführte Begründungssprache zu unterlaufen. Der Botenbericht ist als institutionelle Sicherung des Erzählten angelegt, indem er einen offiziellen Rahmen vor der lantschaft ( ${ }^{\star} \mathrm{B}$ V. 3723), den Führungspersonen des Reiches, erhält. So kann seine Authentizität durch die ,Ohrenzeugen“ „bei späterer Gelegenheit wiederum [...] bezeugt werden“ “. ${ }^{59}$ Als memoria geht er in das kollektive Gedächtnis des burgundischen Herrschaftsverbandes ein. Damit lenkt die Nibelungenklage „das Erzählte in den Kontext mittelalterlicher Memorialkultur zurück.“60

58 Vgl. Swämmels Ordnung vortäuschende Aussage: niemen ich dâ gesehen hân, / der in [i. e. den Burgunden] trüege deheinen haz ( $\left.{ }^{\star} \mathrm{B} \mathrm{V} .3034 \mathrm{f}.\right)$.

59 MÜLLER, Der Spielmann erzählt, S. 94 (Anm. 54).

60 MÜLlER, ,Episches‘ Erzählen, S. 157 (Anm. 11). 
Der Beginn von Swämmels Rede bietet programmatisch einen evaluativen Kommentar des Erlebten:

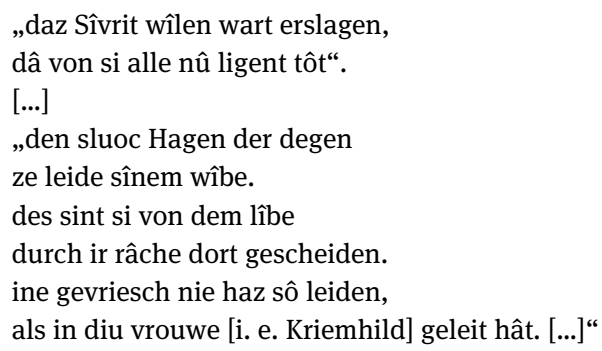

(*B V. 3778-3787)

Der von Hagen verübte Mord an Siegfried fungiert zwar als auslösendes Moment, gleichwohl wird durch das Temporalitätsadverb wîlen eine Zeitlichkeitsstruktur angezeigt, welche die Tat als längst vergangen erscheinen lässt; auf den aktuellen Ort von Kriemhilds Rache, die weitaus mehr Menschen in den Tod gerissen habe, ${ }^{61}$ wird mittels des Lokaladverbs dort verwiesen. Der sich direkt anschließende Unbeschreiblichkeitstopos potenziert Kriemhilds Movens, den haz: Mîn sin der krefte niht enhât, / daz ich ez iu wol künne sagen ( ${ }^{\star} \mathrm{B}$ V. 3788f.). Ein „kategorialer Unterschied zwischen den Erzählungen der beiden Erzähler und dem, wovon sie erzählen“, ${ }^{62}$ ist unverkennbar. Im Rahmen der erzählten Geschichte repräsentiert Swämmels Bericht einen pragmatischen Akt der Wahrheitssicherung im Erzählen und fungiert gleichzeitig als Setzung eines alternativen Deutungshorizonts.

\section{Zwischenresümee}

Die auf unterschiedlichen Ebenen verhandelte Frage nach den Kausalitäten des Nibelungenuntergangs sucht die erzählte irritierte Gesellschaftsordnung durch sinnstiftende christliche Denkkategorien der Zeit aufzuheben und zu stabilisieren; die präsentierte Ordnung des Erzählens basiert auf dem Typus des Planctus, der eine spezielle Möglichkeit von perspektivischer Vervielfältigung bereitstellt, die im Kern zu pluralen Antworten führt. Diese Struktur bietet einen narrativen Reflexionsrahmen für das übergreifende Modell der erzählten Ordnung, deren Eindeutigkeit infolge der unterschiedlichen Informationsvergabe über die vorge-

61 An einer Stelle ist von vierzec tûsent man ( ${ }^{\star} \mathrm{B}$ V. 237) bzw. drîzec tûsent man $\left({ }^{\star} \mathrm{C}\right.$ V. 255) die Rede.

62 Martínez/SChEFFEL, Erzähltheorie, S. 78 (Anm. 57). 
nommenen Fokalisierungswechsel zunehmend infrage gerät, indem differierende Sichtweisen auf das Nibelungengeschehen implementiert werden. Der durch die Erzählerstimme fokalisierte, auf die Entproblematisierung der triuwe zielende evaluative Kommentar wird stellenweise unterlaufen, so dass sich Differenzen zwischen den erzählten Figurenhorizonten und der durch die Erzählerstimme präsentierten Gesamtdeutung auf die triuwe hin ergeben. Der Werte- und Deutungsrahmen scheint zwar vordergründig durch die Erzählerstimme normativ gesichert, die programmatisch die ,richtige“ Lesart des Textes vorgibt und damit gewissermaßen ordnungsstiftend wirkt. Gleichwohl nimmt sie keine Hierarchisierung der Figurenstimmen vor, die den zorn und haz als konkurrierende Begründungsmuster einspielen und damit die Ordnungsfunktion der triuwe umakzentuieren. Weil die Figuren keine Einsicht in die vom Erzähler postulierte Tugendhaftigkeit Kriemhilts haben, erscheinen sie als „Subjekte der Erfahrung der erzählten Welt“, 63 deren divergierende Sichtweisen die „Abhängigkeit normativer Urteile von Standpunkten“ abbilden. ${ }^{64}$ Insofern sich keine Privilegierung einer bestimmten Position oder eine Interaktion zwischen Erzählerstimme und Figurenstimmen erkennen lässt in dem Sinne, dass die Erzählung am kognitiven Horizont einer Figur ausgerichtet wäre, evoziert die Wahl unterschiedlicher Fokalisierungsinstanzen den Effekt eines internen Gesprächs, in dem „divergente Standpunkte $\mathrm{zu}$ Wort kommen, die jeder für sich eine subjektive Berechtigung beanspruchen können“. ${ }^{65}$ So wird das durch den Erzähler grundgelegte ethische Gesamtmodell durch alternative Perspektiven ergänzt und die Notwendigkeit einer produktiven Aushandlung der geltenden Ordnungsvorstellungen, die nicht einfach die eine ,historische Wahrheit' reproduzieren, narrativ entfaltet.

\section{Historische Diskursordnung: Fassungsdivergenz}

Die Klage-Fassungen ${ }^{\star} \mathrm{B}$ und ${ }^{\star} \mathrm{C}$ repräsentieren historisch differente Textzustände, ${ }^{66}$ deren Entstehung in räumlicher und zeitlicher Nähe mit der Verschriftlichung des Nibelungenliedes anzusetzen ist. ${ }^{67}$ Die übereinstimmende Gruppierung von Lied- und Klage-Fassungen dokumentiert einen „dichte[n]

63 HÜBNER, Erzählform, S. 75 (Anm. 30).

64 Ebd., S. 76.

65 MÜlLER, ,Episches‘ Erzählen, S. 201 (Anm. 11).

66 Zum Konstrukt „gleichwertige[r] Parallelversionen“ vgl. STACKMAnN, Karl: Mittelalterliche Texte als Aufgabe. In: Festschrift für Jost Trier zum 70. Geburtstag. Hrsg. von Werner FoERSTE/ Karl Heinz BoRCK, Köln, Graz 1964, S. 241-267, hier S. 263.

67 Bumke, Die vier Fassungen, S. 90 (Anm. 12). 
Prozeß literarischen Umgestaltens“, ${ }^{68}$ in dem die Klage schon vor der Aufspaltung der Überlieferung in die unterschiedlichen Redaktion mit dem Lied verbunden gewesen sein dürfte. ${ }^{69}$ Dass die eigenständige, stärker zur „Verdeutlichung und Erläuterung ${ }^{* 70}$ neigende Akzentuierung der Nibelungenlied-Fassung ${ }^{\star} \mathrm{C}$ sich eng mit dem „motivierend gestaltende[n] und deutende[n] Konzept der Klage“ berührt, ${ }^{71}$ spricht dafür, sie als unmittelbare Reaktion auf das Lied zu sehen und ihre divergierenden Fassungen als Teil eines „,Gesprächs‘ über das aktuelle Erzählen von Heldensage “. ${ }^{72}$ Denn offenbar erschienen die unterschiedlichen Perspektiven auf das Nibelungen-Geschehen in der Klage-Redaktion ${ }^{*} \mathrm{~B}$ als korrekturbedürftig: Die Klage-Fassung ${ }^{\star} \mathrm{C}$ bietet als literarische Anschlusskommunikation an Nibelungenlied und ${ }^{\star} \mathrm{B}$-Klage eine andere Akzentuierung des Bewältigungsnarrativs und dokumentiert damit eine zeitgenössische Diskursordnung. ${ }^{73}$ Insofern kann der Fassungsvergleich in diesem einzigartigen Überlieferungsverbund historische Signifikanz beanspruchen. Im Folgenden wird die Perpetuierung des internen Gesprächs, die bestimmte Positionen fokussiert, verzeichnet, um exemplarisch zu prüfen, ob die Fassung ${ }^{\star} \mathrm{C}$ tatsächlich endgültig „klare (schwarz-weiße) Verhältnisse“"74 schafft.

Markant ist die andersartige Begründung für Siegfrieds Tod: Anders als in ${ }^{\star} \mathrm{B}$ wird die Schuld nicht dem Helden selbst (von sîner übermuot ${ }^{\star} \mathrm{B}$ V. 39), sondern anderen zugewiesen: daz er selbe den tôt / gewan von ander liute übermuot $\left({ }^{\star} \mathrm{C}\right.$ V. $48 \mathrm{f}$.) ${ }^{75}$ Es schließt sich ein kleiner Exkurs über Siegfrieds Vortrefflichkeit an $\left({ }^{\star} \mathrm{C}\right.$ V. $\left.50-72\right)$; darin wird das auf niedrigen Motiven basierende Handeln der Mörder implizit verurteilt, indem die sonst Kriemhilt mit dem haz zugeschriebene affektive Haltung auf ihre Antagonisten verschoben wird: ${ }^{76}$ alsô noch vil maniger tuot, / der guoten liuten traget haz $\left({ }^{\star} \mathrm{C}\right.$ V. 50 f.). Diese Plusverse gegenüber der Fassung ${ }^{\star} \mathrm{B}$ zeigen ein Element kausaler Motivierung an, das sinnstiftend mit der vom Erzähler etablierten triuwe-Ordnung verknüpft ist.

68 HENKEL, Nikolaus: Die Nibelungenklage und die ${ }^{\star} \mathrm{C}$-Bearbeitung des Nibelungenliedes. In: Die Nibelungen. Sage - Epos - Mythos. Hrsg. von Joachim HeINZLE, Wiesbaden 2003, S. 113-133, hier S. 116.

69 BumKe, Die vier Fassungen, S. 541f. (Anm. 12).

70 Ebd., S. 343.

71 Henkel, ${ }^{\star}$ C-Bearbeitung, S. 126 (Anm. 68).

72 Ebd., S. 113.

73 Die Spezifika der Nibelungenlied- und Klage-Fassung ${ }^{\star} \mathrm{C}$ sind herausgearbeitet bei SCHMID, Fassung ${ }^{\star} \mathrm{C}$ des Nibelungenlieds (Anm. 55).

74 LIENERT, Nibelungenklage, S. 24 (Anm. 26).

75 „An keiner anderen Stelle gehen die beiden Hauptfassungen der ,Klage‘ in der Bewertung des Geschehens so weit auseinander wie hier.“ BumKE, Die vier Fassungen, S. 514 (Anm. 12).

76 Ich danke Daniela Fuhrmann für diesen Hinweis. 
Diese nämlich wird im triuwe-Kommentar der Fassung ${ }^{\star} \mathrm{C}$ im ersten Teil des Textes zugleich amplifiziert und gesteigert: Jemand, der einen anderen Menschen zur Hölle verdamme, mache sich der Anmaßung, Gottes Pläne zu kennen, schuldig und lade Sünde auf sich $\left({ }^{\star} \mathrm{C}\right.$ V. $\left.556-566\right) .{ }^{77}$ Weil das Schicksal das „unergründliche Handeln Gottes an und mit dem Menschen“78 exponiere (swie rein er sî und swie guot, / wer weiz, waz got mit im tuot? ${ }^{\star} \mathrm{C}$ V. $567 \mathrm{f}$.), sei auch Kriemhild nicht zu verurteilen, denn:

swaz iemen hât begân, dannoch ist gotes genâden mê, danne iemen sünden begê.

$\left({ }^{\star} \mathrm{C}\right.$ V. 570-572)

In diesem Kontext spielt der Erzähler Kriemhilds triuwe zu Siegfried wieder ein und nimmt damit indirekt auf den eben genannten Exkurs Bezug, der als kohärenzstiftende Legitimierungsfunktion dient:

Sît si mit grôzem jâmer ranc und si grôz triuwe jâmers twanc, die si truoc nâch ir lieben man, als wir von ir vernomen hân, daz si pflac grôzer riuwe durch liebe und durch ir triuwe, daz si zwô sêle und ein lîp wâren, dô si was sîn wîp, dâ von si von schulden zam der râche, die si umbe in nam [...].

$\left({ }^{\star} \mathrm{C}\right.$ V. 573-82)

Eine ähnliche Tendenz zur Umakzentuierung des Kriemhild-Bildes lässt sich auch bei der Figurenfokalisierung beobachten: Hildebrand wiederholt die von der Erzählerstimme eingeführte Aussage, es sei Kriemhilds Absicht gewesen, allein Siegfrieds Mörder zu töten:

daz si in [i. e. Hagen] vor manigen tagen vil gerne het gevrümt erslagen, möhtez si gevüeget hân.

$\left({ }^{\star}\right.$ C V. 1309-1311)

77 von welchem urkünde / mac er des gewisheit hân, / sô der mensche hie muoz lân / daz leben, war er danne var? / sich selben niemen alsô gar / sol unschuldec machen / von süntlîchen sachen. ( ${ }^{\star} \mathrm{C}$ V. 560-566).

78 HeNKEL, Nibelungenlied und Klage, S. 93 (Anm. 21). 
Diese Stelle fehlt in der Fassung *B ebenso wie die Zuschreibung der Schuld an Bloedelin, die die Verantwortung für den Ausbruch der Kämpfe von Kriemhild ablenkt: Ohne seinen explizit als unsin $\left({ }^{\star} \mathrm{C}\right.$ V. 1338) bezeichneten Angriff auf die burgundischen Knechte hätte es kein Gemetzel gegeben: het Bloedelîn eine den strît vermiten, / sô waere niht übels hie geschehen ( ${ }^{\star} \mathrm{C}$ V. 1342f.).

Gleichwohl ist die Informationsvergabe durch die Fokalisierungsinstanzen (Erzähler- und Figuren) in Fassung ${ }^{\star} \mathrm{C}$ partiell inhomogen; ein kleinteiliger Vergleich offenbart Uneinheitlichkeiten gegenüber den übergeordneten Tendenzen. Vier Beispiele:

Die Erzählerstimme schildert in Fassung ${ }^{\star} B$, wie Kriemhild ihre Rache erst ins Werk setzt, nachdem sie sich der Unterstützung durch das hunnische Gefolge sicher fühlt ( ${ }^{\star} \mathrm{B}$ V. 82-87); ihre Pläne erfolgen dabei âne undersprâche ( ${ }^{\star} \mathrm{B}$ V. 99) - ein Aspekt, den in ${ }^{\star} \mathrm{C}$ die Wendung vil tougenlîchen ( ${ }^{\star} \mathrm{C}$ V. 126f.) intensiviert; der folgende Vers, die si doch sît volbrâhte $\left({ }^{\star} \mathrm{C}\right.$ V. 128), markiert Kriemhilds alleinige Verantwortlichkeit, als deren verheerendes Resultat viele tapfere Männer das Leben gelassen haben ( ${ }^{\star} \mathrm{C}$ V. $130 \mathrm{f}$.).

Bemerkenswerterweise wird ausgerechnet in Fassung ${ }^{\star} \mathrm{B}$ eine Änderung von Kriemhilds Todesart vorgenommen: von der Zerstückelung im Lied (Str. 2377) zur Enthauptung in der Klage ( ${ }^{\star}$ B V. 795-798), die die Strafe der Verräter zu einer Form der Hinrichtung um-ordnet, „die Privileg des Vornehmen ist und seine Ehre nicht tangiert" ${ }^{79}$ Dieser Aspekt bleibt in der Fassung ${ }^{\star} \mathrm{C}$ an der entsprechenden Stelle unerwähnt.

Etzel äußert in seiner Klagerede um Kriemhild offenkundige Kritik an deren Racheplan:

waere Hagen alterseine erslagen, daz waere ein guot list gewesen. sô waeren die andern genesen, die nû hie ligent verschrôten.

$\left({ }^{\star}\right.$ C V. 2016-2019)

Dietlinds bereits zitierte Anklage, es sei nicht gut gewesen, dass jemand, der triuwe haben wolde ( ${ }^{\star} \mathrm{B}$ V. 3192), Gernot und Rüdiger mit râte ( ${ }^{\star} \mathrm{B}$ V. 3191) gegeneinander aufgebracht habe, kommt in der Fassung ${ }^{\star} \mathrm{C}$ zwar zunächst ohne den Hinweis auf die triuwe aus:

ez was ein michel schande, daz man zwischen in beiden den strît niht kunde scheiden.

$\left({ }^{\star}\right.$ C V. 3312-3314)

79 MÜLLER, Spielregeln, S. 168 (Anm. 39). 
Gleichwohl fokussiert die Figurenstimme ausdrücklich Kriemhilds untriuwe; ein Akzent, der sich in ${ }^{\star} \mathrm{B}$ nicht findet:

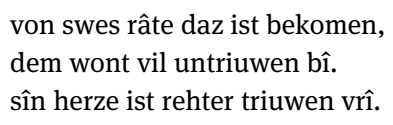

${ }^{\star}{ }^{\star}$ C V. 3318-3320)

Die Bekräftigung dieses Vorwurfs durch Swämmel, daz tet, vrouwe, niemen mêr / wan der küneginne lîp ( ${ }^{\star} \mathrm{C}$ V. 3322f.), erscheint geradezu als Kontrast gegen die von der Erzählerstimme zu Beginn der Dichtung eingeführte triuwe-Deutung.

Es lässt sich festhalten, dass die Klage-Fassung ${ }^{\star} \mathrm{C} \mathrm{zu}$ einer spezifischen Akzentuierung des Erzählten tendiert, die eine historische Diskursordnung mit dem Ziel höherer Kohärenzstiftung, Motivierung und Deutung der Handlung dokumentiert, indem die in ${ }^{\star} \mathrm{B}$ dominierenden unterschiedlichen Standpunkte der Figurenstimmen mit der Erzählerstimme tendenziell harmonisiert werden. Dennoch bleibt das Kriemhild-Bild stellenweise ambivalent, da die Fokalisierungsinstanzen keine definitiv ,verbindliche“ Einstimmigkeit erkennen lassen. Dass eine solche alternative Organisation der Erzählperspektiven in der Anschlusskommunikation an das Nibelungenlied in den Klage-Fassungen für die zeitgenössischen Rezipienten als notwendig empfunden wurde, zeigt die Klage-Fassung *J an, die dieses ,Desiderat' allerdings nur durch eine grundsätzlich geänderte Konzeption und eine radikale Kürzung des Textes um etwa drei Viertel seines Umfangs erreicht. ${ }^{80}$ Die weitere Rezeption des Kriemhild-Bildes im Spätmittelalter hingegen zeigt, dass der in den Redaktionen der Klage greifbare Versuch einer Entlastung und Sympathielenkung hin zu Kriemhild offenbar wenig Erfolg hatte. ${ }^{81}$

80 Zum Kürzungsverfahren der *J-Klage vgl. FRICK, Julia: abbreviatio. Zur historischen Signifikanz von Kürzungsfunktionen in der mittelhochdeutschen höfischen Epik des 13. Jahrhunderts. Eine Projektskizze. In: PBB 140.1 (2018), S. 23-50. Siehe auch umfassend KIEHL, Christina: Zur inhaltlichen Gestaltung einer Kurzfassung. Eine verkürzte `Nibelungen-Klage` als Fortsetzung des `Nibelungen-Liedes`, Frankfurt a. M. 2008 (Hamburger Beiträge zur Germanistik 45).

81 In der Nibelungenlied-Hs. $\mathrm{n}$ wird Kriemhild als „streit- und rachsüchtige Königin“ inszeniert und erhält die „negativste[n]“ Konturen „innerhalb der >Nibelungenlied<-Überlieferung“. KOFLER, Walter: Das Nibelungenlied n. In: PBB 136 (2014), S. 76-120, hier S. 113 u. 118. 


\section{Erzählte Ordnung und Ordnung des Erzählens in der Nibelungenklage}

Die Nibelungenklage bildet als erstes Rezeptionszeugnis des Nibelungenliedes „den übergeordneten Bezugsrahmen “82 für die Einordnung des Erzählten in die Kategorien von Schuld und Sühne als „Formen christlicher Leidbewältigung“. ${ }^{83}$ Die Un-Ordnung, die sich am Ende des Nibelungenliedes aufgetan hat, wird in die erzählte Ordnung der christlichen Heilserwartung nach dem Tod überführt, wie es die Auferstehung Christi symbolisiert (et sicut in Adam omnes moriuntur ita et in Christo omnes vivificabuntur, 1 Cor 15,22): ${ }^{84}$ Von der Überwindung des Todes im verklagen bis zur Stiftung der Herrschaft und damit der Restitution des ordo durch die Krönung von Gunthers und Brünhilds Sohn Siegfried. ${ }^{85}$

Diese erzählte Ordnung wird auf unterschiedlichen Ebenen zur Ordnung des Erzählens ins Verhältnis gesetzt und mittels perspektivischer Vervielfältigung der Stimmen organisiert: Die Erzählerstimme etabliert zwar eine programmatische Deutung, indem das Motiv der triuwe als Begründungssprache für Kriemhilds Handeln eine Ordnungsfunktion für die in Figurenreden vorgetragenen Kommentare übernimmt; gleichwohl korreliert diese nicht einfach mit der durch andere Fokalisierungsinstanzen vorgenommenen Informationsvergabe. Diese konstituieren vielmehr als Repräsentanten einer Diversität von Weltsichten auf das Erzählte eine „plurale, in unterschiedliche individuelle Ansichten aufgesplitterte Erzählwelt“"86 und damit ein anders akzentuiertes Bewältigungsnarrativ. Weil die Erzählerstimme weder eine Hierarchisierung vornimmt, noch einen Figurenstandpunkt privilegiert, kommen divergierende Perspektiven nebeneinander zum Stehen, in denen sich verschiedene ,Antworten“ im Rahmen eines internen Dialogs über den Nibelungenuntergang herauskristallisieren. Insbesondere der eine metadiegetische Dimension eröffnende Botenbericht Swämmels als Spiegelung der Erzählerfunktion präsentiert eine konkurrierende Perspektive von Welterleben.

Die Abkehr vom Paradigma konzeptionell mündlichen Erzählens geht in der Nibelungenklage einher mit einem Rückgriff auf genuin buchepische Darstel-

82 HENKEL, Nibelungenlied und Klage, S. 75 (Anm. 21).

83 Henkel, ${ }^{\star}$ C-Bearbeitung, S. 120 (Anm. 68). Das Geschehen wird auf Gottes Handeln mit der Welt zurückgeführt: got der woldes in niht geben, / daz in daz liep geschaehe, / daz in deheiniu saehe / bî gesundem sînem lîbe ( ${ }^{\star} \mathrm{B}$ V. 1942-1945).

84 „Denn wie alle in Adam sterben, so werden alle in Christus lebendig gemacht werden.“

85 Dass Gunthers und Brünhilds Sohn durch des heldes liebe den Namen Siegfried bekommen hat, berichtet das Nibelungenlied (Str. 718). In der Klage fällt der Name nicht.

86 MÜlLER, ,Episches‘ Erzählen, S. 198f. (Anm. 11). 
lungsmodi, die eine auf das jeweilige Figurenwissen rekurrierende Aushandlung des vom Erzähler gesetzten Deutungsrahmens über Fokalisierungen spezifischer narrativer Instanzen ermöglichen. Insofern wäre die Narration in der Nibelungenklage (jedenfalls in Bezug auf die Fassung ${ }^{\star} \mathrm{B}$ ) explizit vom ,epischen“ Erzählen, in dem es - zugespitzt - „nur die eine, die ,richtige“ Version“ ${ }^{87}$ geben kann, zu unterscheiden. Das durch den Typus des Planctus ermöglichte perspektivische Erzählen entfaltet ein diffiziles Panorama teils ineinandergreifender, teils einander kontrastierender Deutungsoptionen, die die erzählte Ordnung vom Nibelungenuntergang mittels der Ordnung des Erzählens verunsichern. Sie fokussieren einen internen ,Kommunikationsprozess', der einen in Ansätzen gleichberechtigten Platz neben dem ethischen Gesamtmodell beanspruchen kann und der auf die generelle Unverfügbarkeit der göttlichen Providenz für die menschliche Ratio verweist.

Dass dieses Konzept wohl schon in unmittelbarem zeitlichen Umfeld offenbar als diskutabel erschien, dokumentiert die Fassungsdivergenz als historische Diskursordnung: Die Klage-Fassung ${ }^{\star} \mathrm{C}$ (und noch signifikanter ${ }^{\star} \mathrm{J}$ ) ${ }^{88}$ bietet eine alternative Perspektivierung des Erzählten, die mittels der Favorisierung eines Standpunktes auf die Profilierung einer einheitlicheren Begründung des Bewältigungsnarrativs zielt. Die erzähltechnisch realisierte Ordnungsdiskussion ,antwortet' dabei auf die Einbettung in den jeweiligen situativen historischen Kontext, sodass in der ${ }^{\star} \mathrm{C}$-Klage eine Aktualisierung des narrativen Integrationspotentials in einen jenseits von Einzelstimmen dominanten ,Sinngebungsprozess` erfolgt, wie ihn die Fassung ${ }^{\star} \mathrm{C}$ des Nibelungenliedes entwirft. ${ }^{89}$ So zeichnet sich in der ${ }^{\star} \mathrm{C}$-Klage eine zwar durch die gleichen Erzählinstanzen fokalisierte, aber durch eine merkliche Reduktion perspektivischer Unterscheidung tendenziell ,eindeutigere“ Lesart ab, die stärker vom Gestus „epische[r] Einstimmigkeit““90 beeinflusst $\mathrm{zu}$ sein scheint.

87 Ebd., S. 211.

88 Im Hinblick auf die Klage-Fassung *J vgl. FRICK, Julia: Reflexionen des Untergangs. Erzählen vom Ende in den Fassungen der Nibelungenklage. In: Die Zeit der Letzten Dinge. Deutungsmuster und Erzählformen des Umgangs mit Vergänglichkeit in Mittelalter und Früher Neuzeit. Hrsg. von Julia WeItBRECHT/Andreas BiHRER/Timo FELBER, Göttingen 2020 (Encomia Deutsch 6), S. $125-142$.

89 Die Ansätze der Forschung zum „Nibelungenkomplex ${ }^{\star} C$ “ zusammenführend ScHmid, Fassung ${ }^{\star} \mathrm{C}$ des Nibelungenlieds (Anm. 55), bes. S. 255-317.

90 MÜLlER, ,Episches` Erzählen, S. 210 (Anm. 11). 